\title{
EL ROL DE LAS “ACTITUDES GLOBALES DISCRIMINATORIAS” EN EL DESARROLLO HUMANO: UNA REFLEXIÓN PSICOSOCIAL DESDE EL FEMINISMO DE LA INTERSECCIONALIDAD
}

\section{Libertad Jiménez Almirante}

libalji@hotmail.com

Centro de Estudos Internacionais do Instituto Universitário de Lisboa (Portugal)

Recibido: 11-10-2014

Aceptado: 23-04-2015

\section{Resumen}

Se ha pretendido tender un puente de unión entre la teoría del desarrollo humano de Amartya Sen (1985) y Martha Nussbaum (2000) y el modelo ecológico del desarrollo humano de Urie Bronfenbrenner (1987 [1979]). El nexo pudiera residir en el hecho de reconocer que el acceso a la riqueza es indispensable para garantizar el pleno desarrollo de las personas pero las barreras para dicho acceso pudieran no ser una cuestión solamente material sino también una cuestión actitudinal originada en los entornos del desarrollo humano. Asimismo, las barreras discriminatorias sufridas por las mujeres son muchas e interseccionadas como apunta Kimberlé Crenshaw (1995) desde el feminismo de la interseccionalidad.

Palabras clave: Desarrollo, mujeres, género, actitud, discriminación, feminismo.

\begin{abstract}
A bridge has been tried to build between the theory of human development of Amartya Sen (1985) and Martha Nussbaum (2000) and the ecological model of human development of Urie Bronfenbrenner (1987 [1979]). The link could be in the fact of recognizing that the access to wealth is essential to ensure the full development of people, but the barriers to such access could be a matter not only material but also an attitudinal question caused in the environments of the human development. In addition, discriminatory barriers suffered by women are many, and they are intersected as Kimberlé Crenshaw (1995) explains from the feminism of intersectionality.
\end{abstract}

Keywords: Development, women, gender, attitude, discrimination, feminism. 


\section{Introducción}

Las perspectivas sobre el desarrollo socioeconómico que han atendido a la óptica de género, principalmente desde el feminismo socialista (Benería y Sen, 1983; Hartmann, 1981; Smith, 1974), han centrado su atención en el análisis del macrosisma (entorno ideológico) y el exosistema (entorno sistémico-funcional) del "sujeto en desarrollo", localizando la óptica atencional, consecuentemente, no tanto en el "sujeto en desarrollo" sino más bien en las dinámicas socioeconómicas de los "países en desarrollo" en un contexto globalizado(r). Es decir, desde estas perspectivas, se han centrado los esfuerzos en el análisis de los sistemas patriarcales y capitalistas, y las ideologías derivadas de ellos, para el examen de las desigualdades de género en el desarrollo desde un punto de vista macrosistémico y exosistémico.

Asimismo, en el seno de la preocupación por resolver una situación a todas luces injusta, con respecto a las mujeres, que tiene que ver con el avance del desarrollo social de los países dejando en el arcén a "ellas" (las mujeres), esta perspectiva se ha centrado en el análisis de los roles de género otorgados respectivamente, por parte de dichos sistemas patriarcal y capitalista, a hombres y a mujeres en las sociedades. Dicha atención pormenorizada sobre los roles de género tiene que ver con la consideración de que en la casuística de las funciones desempeñadas por hombres y mujeres reside el problema sobre tener, o no tener, acceso y control con respecto a los recursos necesarios para llegar a conseguir una vida digna. Sin embargo, fiar todos los esfuerzos a la transformación de los roles de género puede que tenga como resultado, efectivamente, una transformación de los papeles asignados socialmente a hombres y a mujeres pero sin la pertinente variación de la "posición" entendida por Kate Young (1988) como la situación inmaterial en la que se encuentran las mujeres con respecto a los hombres en una determinada sociedad.

En este sentido, puede que en el proceso de transformación de los roles de género las mujeres pasen al plano de la producción, si es que antes se encontraban sólo en el plano de la reproducción aunque ya se ha comprobado que esto no es todas las veces cierto porque existe una dificultad manifiesta para contabilizar el trabajo productivo que habitualmente realizan las mujeres (Durán, 2012; Ward y Pyle, 1995), pero la no localización del foco atencional en la transformación de los esquemas mentales de género (con estereotipos y prejuicios creados concienzudamente en contra de las mujeres) si es a un nivel personal (Bem, 1981) o en la 
transformación del imaginario colectivo creado socialmente sobre los géneros (con estereotipos y prejuicios sociales creados concienzudamente en contra de las mujeres) si es a un nivel social (Sastre y Moreno, 2004) hace que las mujeres terminen ocupando lugares en la producción considerados sociolaboralmente "inferiores" (además de menos productivos, menos reconocidos socialmente, menos remunerados, menos seguros laboralmente, etc.) que limitan su pleno desarrollo sin que esto suponga el abandono de una posición social inferiorizada. Es decir, a juicio propio, el desarrollo no únicamente puede analizarse desde un punto de vista macrosistémico o exosistémico en relación al funcionamiento del patriarcado y el capitalismo sino que también se trata de una cuestión mesosistémica y microsistémica que se encuentra relacionada con cómo son consideras (o percibidas) psicosocioculturalmente las mujeres con respecto a los hombres, es decir, qué es aquello que se piensa con respecto a "ellas" (las mujeres) en contraposición a lo que se piensa con respecto a "ellos" (los hombres) siguiendo los postulados de Simone de Beauvoir (2008 [1949]).

Detrás de una serie de preguntas acerca de por qué las mujeres son impedidas a acceder a su desarrollo como personas sujetas a dicho derecho declarado en la Cuarta Conferencia Mundial sobre la Mujer (Organización de las Naciones Unidas, 1996), celebrada en Beijing en el año 1995, como, por ejemplo, por qué las mujeres ocupan un menor número de cargos de responsabilidad en las empresas o por qué las mujeres son consideras mercancía susceptible de ser explotada sexualmente, podrían existir respuestas microsistémicas y mesosistémicas (además de exosistémicas y macrosistémicas). Es decir, detrás de esas preguntas debería haber una respuesta, además de la respuesta que tiene que ver con asegurar que esos hechos se dan porque existe un sistema patriarcal y capitalista que propicia el impedimento del desarrollo de las mujeres o la violación de sus derechos más elementales, que tiene que ver con responder: porque la gente tiene una forma determinada de pensar acerca de lo que es "ser mujeres" y lo que es "ser hombres" y esa forma de pensar puede llegar a determinar aquello que "las mujeres pueden llegar a ser" socialmente admitido y aquello que "los hombres pueden llegar a ser" socialmente admitido.

Toda vez que han sido analizados los resultados y conclusiones obtenidos a partir de una investigación de doctorado de cuatro años de duración, que versa sobre la utilización transversal de la perspectiva de género en los proyectos de cooperación internacional para el desarrollo (dicho trabajo ha supuesto el análisis de 259 proyectos financiados por la Agencia Española de Cooperación Internacional para el Desarrollo - AECID y se ha contado con la 
colaboración de 16 técnicos/as de las Organizaciones No Gubernamentales dedicadas al Desarrollo - ONGD y 12 técnicos/as de AECID, que han respondido a un cuestionario online con preguntas abiertas, y con la contribución de protagonistas locales de las acciones de desarrollo que han participado en 16 grupos de discusión realizados en Ecuador, Mozambique y Senegal) se ha considerado conveniente realizar una propuesta de análisis sobre la conformación del desarrollo del ser humano, desde un punto de vista psicosociocultural, teniendo en cuenta el modelo ecológico del desarrollo humano de Urie Bronfenbrenner (1987 [1979]). Dicha propuesta se planteó tras la necesidad de responder a dos interrogantes puestos de manifiesto: por qué las desigualdades de género son la única constante manifiesta en todos los grupos de discusión realizados y por qué las mujeres sufren en mayor medida una serie de discriminaciones que "debieran" afectar de igual modo también a los hombres.

De forma tentativa, a juicio propio, una de las posibles respuestas a los interrogantes planteados podría residir en la consideración de que el desarrollo relativo al ser humano también depende de una serie de "actitudes globales discriminatorias" (que terminan por convertirse en "actitudes globales expropiatorias de derechos") y, además, de la necesaria generación de condiciones óptimas económicas y políticas que propicien el desarrollo de lo que Amartya Sen (2000 [1999]) denomina la "agencia personal del individuo". Es decir, el desarrollo humano no sólo debería definirse, en términos de comprensión o sensibilidad con los infortunios ajenos en cuanto al acceso a la riqueza necesaria para el desarrollo vital, como "un proceso mediante el cual se amplían las oportunidades de los individuos, las más importantes de las cuales son una vida prolongada y saludable, acceso a la educación y el disfrute de un nivel de vida decente [...]" (Programa de Naciones Unidas para el Desarrollo, 1990: 33) sino, también, debería definirse, en términos de aquel desarrollo perteneciente al ser humano, como "cambio perdurable en el modo en que una persona percibe su ambiente y se relaciona con él" (Bonfenbrenner, 1987 [1979]: 23).

La justificación de por qué resulta pertinente dicha propuesta viene determinada por dos razones fundamentalmente. Por un lado, tras la realización de la investigación, ha quedado constatada la necesidad de añadirle un valor transformador a la utilización de la perspectiva de género en el contexto de las acciones de cooperación internacional para el desarrollo más allá de la mera consideración retórica actual y, además, dicha transformación pasa por eliminar una serie de actitudes globales que dificultan el desarrollo, principalmente, de las mujeres. Por otro lado, los grupos de discusión han puesto de manifiesto que las opresiones sufridas por parte de 
algunas mujeres van más allá del sistema de socialización de género, aunque dichas otras opresiones también coexistan con este sistema.

Por lo tanto, se va a intentar justificar por qué el desarrollo del ser humano, también en el contexto del desarrollo socioeconómico, debe ser considerado una cuestión de actitud que implica dos tipos de actitudes fundamentalmente: "actitudes globales discriminatorias" o "actitudes globales expropiatorias de derechos". Unas actitudes que, por otro lado, son adquiridas por los sujetos (proceso psicosocial) y propagadas a través del proceso de socialización global del que son protagonistas.

Que conste, por adelantado, la asunción, por un lado, de los postulados fundamentales del enfoque de las capacidades básicas (Sen, 1985, 1990; Nussbaum, 2000) teniendo en cuenta que la riqueza y el desarrollo son dos herramientas fundamentales para conseguir la felicidad de los individuos con una base profunda en la consecución de su libertad; por otro lado, la asunción del enfoque de los derechos humanos (Cruz De la, 2007), teniendo en cuenta que el desarrollo pasa a ser considerado un derecho y una meta que persigue el cumplimiento de los derechos humanos fundamentales; asimismo, la asunción del enfoque de género y desarrollo/ enfoque de género en el desarrollo (Sen y Grow, 1987; Whitehead, 2006 [1979]), que toma como objetivo la eliminación de las desigualdades de género que impiden el pleno desarrollo humano de las mujeres para la consecución de aquellos que son también sus derechos fundamentales; de igual modo, la asunción de los postulados del feminismo de la igualdad (Amorós, 1992; Firestone, 1976 [1970]; Lerner, 1990 [1986]; Millett, 1970), que considera el patriarcado como un sistema de opresión contra las mujeres que es universal y transversal a todas las otras formas de opresión como pueden ser la etnicidad o la clase social y, por último, la asunción de los fundamentos del feminismo de la interseccionalidad (Anzaldúa, 2004 [1987]; Collins, 2000; Crenshaw, 1995; Davis, 2005 [1981]; hooks, 1984; Sánchez Néstor, 2005) que considera que las opresiones ejercidas en contra de las mujeres para el impedimento de su desarrollo son muchas e interseccionadas. 


\section{Propuesta de análisis sobre el rol de las actitudes frente al desarrollo humano promulgado por las acciones de desenvolvimiento internacional}

La propuesta de análisis realizada sobre el rol de las actitudes frente al desarrollo humano promulgado por las acciones de desenvolvimiento internacional viene determinada, a continuación, por dos aspectos fundamentales: por un lado, la definición de los conceptos "actitudes globales discriminatorias" y "actitudes globales expropiatorias de derechos" y, por otro lado, la descripción de la conformación de las "actitudes globales discriminatorias" mediante el modelo ecológico del desarrollo del ser humano.

\subsection{Definición de los conceptos "actitudes globales discriminatorias" y "actitudes globales expropiatorias de derechos" y su implicación en las acciones para el desarrollo}

Para Kimball Young (1967), las actitudes son predisposiciones aprendidas que tienen como consecuencia la respuesta por parte de un determinado individuo a reaccionar de una forma positiva o negativa, más o menos consecuente consigo mismo, ante un determinado hecho, idea, persona, cosa, etc. Al respecto, hace casi cincuenta años, Kimball Young (1967) se planteaba dos cuestiones fundamentales que van a servir de punto de partida para definir el concepto "actitudes globales discriminatorias”: (1) ¿Existen, en efecto, constelaciones de actitudes? y (2) ¿Qué relación puede establecerse entre el concepto de actitud y el de representación colectiva? En este sentido, por un lado, las "actitudes globales discriminatorias" podrían definirse como aquellas predisposiciones aprendidas y adoptadas por un grupo de personas, más o menos numeroso, que han sido divulgadas y arrogadas mundialmente (sin limitación de fronteras) y que comparten como rasgo común la discriminación entre dos o más grupos de personas, otorgando la actitud positiva frente al grupo (o grupos) percibido (o percibidos) de mayor afinidad y reservando la actitud negativa frente al grupo (o grupos) percibido (o percibidos) de menor afinidad. Por otro lado, las "actitudes globales expropiatorias de derechos" podrían considerarse aquellas predisposiciones aprendidas y adoptadas por un grupo de personas, más o menos numeroso, que han sido divulgadas y arrogadas mundialmente ( $\sin$ limitación de fronteras) y que comparten como rasgo común la discriminación entre dos o más grupos de personas, otorgando la actitud positiva (sujeta a derecho) frente al grupo (o 
grupos) percibido (o percibidos) de mayor afinidad y reservando la actitud negativa (no sujeta a derecho) frente al grupo (o grupos) percibido (o percibidos) de menor afinidad.

El grupo de personas que comparte esa determinada actitud discriminatoria se podría definir en los términos que propone Benedict Anderson (1993 [1983]), en el contexto de sus estudios sobre los nacionalismos, como "comunidad imaginada". Dicha comunidad es, además, segregacionista y tiene como peculiaridad que quizás no lleguen a conocerse todos los miembros de la colectividad que comparten dicha actitud discriminatoria pero, al mismo tiempo, se profesan un compañerismo sin parangón allá donde estén en cualquier parte del mundo.

Las actitudes son fundamentales en la concepción del desarrollo de las personas porque hasta el hecho de decidir, desde la política económica, cómo emplear las inversiones económicas destinadas al desarrollo es una cuestión también actitudinal-gubernamental. La pobreza, y especialmente la feminización de la pobreza (Espino, 2007; Paredes, 2012), se encuentran perpetuadas por la falta de acceso a los recursos imprescindibles para la consecución de una vida digna por parte del "sujeto en desarrollo" pero dicho acceso no viene determinado simplemente por el hecho de no haber o no existir (en el contexto de desarrollo de las personas) recursos disponibles a los que poder acceder sino que viene determinado, también, por el hecho de ser impedidos a acceder a una serie de recursos que de no existir dicho impedimento sí se encontrarían disponibles. En este sentido, el término "pobreza" podría definirse como el resultado del padecimiento, por parte de una persona o conjunto de personas, de una, o varias, de las "actitudes globales discriminatorias" (o "actitudes globales expropiatorias de derechos") proyectadas, a su vez, por otra persona o un conjunto de éstas.

Dicho impedimento sobre el acceso al desarrollo podría ser llevado a cabo mediante la perpetuación de una serie de actitudes que, por un lado, podrían encontrarse fundamentadas ideológicamente, por ejemplo, en el androcentrismo (Gilman, 2011 [1911]), el racismo (Collins, 2000), la xenofobia (Anzaldúa, 2004 [1987]), el clasismo (Benería y Sen, 1983), el etnocentrismo (Sánchez Néstor, 2005) o la homofobia (Ottosson, 2007) y, por otro lado, podrían encontrarse articuladas respectivamente, a un nivel sistémico, en torno al patriarcado (Amorós, 1992), el capitalismo (Hartmann, 1981), el apartheid o la esclavitud (Davis, 2005 [1981]), la globalización en su vertiente más depredadora (Cobo, 2010), la heteronormatividad (Witting, 2006 [1992]; Butler, 2006 [2004]) o el nacionalismo (Anderson, 1993 [1983]). El denominador común entre dichas ideologías y sus correspondientes sistemas de 
funcionamiento es la negación (de una forma cognitiva, afectiva y conductual) del principio fundamental de igualdad entre los seres humanos a través de la consideración de “inferioridades humanas" (entendidas éstas como "humanidades inferiorizadas", es decir, humanidades sobre las que se ha ejercido objetivamente la acción de darles un valor inferior de una forma antinatural) y "superioridades humanas" (entendidas éstas como "humanidades superiorizadas", es decir, humanidades sobre las que se ha ejercicio objetivamente la acción de darles un valor superior de una forma antinatural) y la perpetuación de dicho denominador común podría llevarse a cabo mediante una serie de "actitudes globales discriminatorias" (o expropiatorias de derechos), respectivamente, de tipo machistas, racistas, xenófobas, clasistas, etnocéntricas u homófobas. Además, dichas actitudes no son compartimentos estancos sino que, por un lado, pueden confluir (o coexistir al mismo tiempo) y, por otro lado, pueden compartir rasgos comunes de los cuales el principal es la acción de discriminación.

Por lo tanto, para romper las barreras que impiden el acceso al desenvolvimiento humano sería necesario integrar en las acciones de desarrollo un programa educativo que tuviera en cuenta un cambio actitudinal que deberá abarcar todos los componentes de las actitudes que señalaba Steven Breckler (1984): componente cognitivo (opiniones, percepciones o creencias), componente afectivo (aspecto emocional) y componente conductual (tendencia a reaccionar).

Asimismo, ese cambio actitudinal requeriría de un examen en profundidad de cada uno de los fenómenos psicosocioculturales y económicos que puedan ser sometidos a análisis (machismo, racismo, xenofobia, clasismo, etnocentrismo, homofobia, etc.) pero, a grandes rasgos, el cambio actitudinal podría pasar, en primer lugar, por la ruptura de una percepción orientada a los seres humanos como ciudadanos/as sustancialmente desiguales (componente cognitivo); en segundo lugar, podría transitar por ultrapasar emocionalmente las evocaciones hacia una supuesta inferioridad o superioridad de los seres humanos (componente afectivo) y, por último, podría pasar por romper con la tendencia a reaccionar, bajo sospecha de parcialidad, en circunstancias que requieren de un trato basado en la igualdad de derechos y oportunidades (componente conductual). El cambio actitudinal requerido, tomando las palabras de Ma Ángeles Durán (1993) en relación al fin del androcentrismo en las ciencias sociales, podría pasar por la transformación de un pensamiento humano hacia lo igualitario (basado en la no discriminación por razón moral, intelectual o física). 
Las actitudes se encuentran ancladas en los esquemas mentales, que son según Sandra Lipsitz Bem (1981), a colación de su teoría sobre los esquemas mentales de género, estructuras del pensamiento (estructuras abstractas del conocimiento) que sirven para, entre otras cosas, organizar la información social que nos rodea con el objetivo de optimizar los esfuerzos para su comprensión y la orientación de nuestra actuación frente a la realidad que vivimos. Asimismo, los esquemas mentales pueden presentar dos elementos estructuradores fundamentalmente: los estereotipos, que según Ester Barberá (2004) son sistemas de creencias compartidas sobre determinados grupos sociales, y los prejuicios, que según Gordon W. Allport (1968 [1954]) son juicios de valor otorgados sobre determinados grupos sociales. La proyección social de los esquemas mentales podría considerarse que es el imaginario colectivo, que según Genoveva Sastre y Montserrat Moreno (2004) consiste en un sistema de pensamiento compartido por un colectivo social que conduce a dar por supuestas una serie de normas, estereotipos y prejuicios implícitos.

En este sentido, las acciones de desarrollo programadas pueden representar dos papeles fundamentales a través de su implicación educativa (indirecta o directamente). Por un lado, una acción de desarrollo correctamente programada puede ser un vehículo para la transformación de las "actitudes globales discriminatorias" a través de la transformación de los imaginarios colectivos machistas (en imaginarios colectivos antimachistas); imaginarios colectivos racistas (en imaginarios colectivos antiracistas); etc. Por otro lado, una acción de desarrollo incorrectamente programada puede ser un vehículo multiplicador de las "actitudes globales discriminatorias" a través de la perpetuación de los imaginarios colectivos machistas (en imaginarios colectivos machistas fortalecidos); imaginarios colectivos racistas (en imaginarios colectivos racistas fortificados); etc. Por último, aunque el objetivo específico de la acción de desarrollo no vaya orientado a la educación, como señalaba Orlando Fals Borda (1959), una acción de desarrollo siempre lleva implícito un programa educativo que pretenderá modificar actitudes.

También es cierto que las acciones de cooperación internacional para el desarrollo, en ocasiones, pueden ir dirigidas específicamente a fomentar el empoderamiento (Schuler, 1997) de determinados grupos objetivos que se ven afectados por dichas "actitudes globales discriminatorias" como, por ejemplo, un grupo de mujeres rurales en Mozambique, un grupo de población afrodescendiente en Ecuador, inmigrantes malienses en Guinea Ecuatorial, un grupo de minoría étnica como los peul en Senegal, un colectivo de homosexuales y 
transexuales en India, etc. Sin embargo, la acción de desarrollo sería más efectiva en la meta de acabar con las "actitudes globales discriminatorias" (o expropiatorias de derechos) si fuesen orientadas a toda la población en lugar de ir dirigida específicamente al grupo discriminado. Se va a intentar argumentar dicha reflexión a través de tres razones fundamentalmente: por un lado, quizás el grupo discriminado pueda adoptar herramientas para autoempoderarse en el camino del desarrollo pero, también, ciertamente, el hecho de no trabajar en el contexto que le rodea va a permitir que siga existiendo una serie de límites (barreras) actitudinales que dificultan concienzudamente su desarrollo; por otro lado, el desarrollo del grupo discriminado no depende sólo de su autoempoderamiento sino que depende, también, de la proyección de su empoderamiento en el contexto que les rodea, es decir, el desarrollo humano debería entenderse, a juicio propio, como una interdependencia entre la transformación "propia" y la transformación del "otro" y, por lo tanto, quizás pudiera entenderse que la transformación requerida para el desarrollo no sólo dependiera del "sujeto en desarrollo" sino, también, de la transformación del contexto donde éste se desarrolla y, por último, el problema sobre el acceso al desarrollo lo padece el grupo discriminado pero las cusas del problema (las "actitudes globales discriminatorias") no residen en el grupo discriminado sino en su entorno.

\subsection{Descripción de la conformación de las "actitudes globales discriminatorias" mediante el modelo ecológico del desarrollo del ser humano}

Asimismo, se ha querido utilizar el modelo ecológico del desarrollo humano de Urie Bronfenbrenner (1987 [1979]) para analizar, desde la perspectiva ecológica del desarrollo de la conducta humana, una hipotética posición de los elementos que intervienen en la perpetuación de las desigualdades humanas, y especialmente en las desigualdades de género, en los diferentes entornos (microsistema, mesosistema, exosistema y macrosistema) que propician el desarrollo de los sujetos. Se expone, a continuación, una interpretación de la posición que pudieran presentar dichos elementos a través de la siguiente Figura 1: 
Figura 1. El modelo ecológico del desarrollo humano frente al origen de las desigualdades humanas

MICROSISTEMA: familia o agregado familiar encargado de la asignación de roles de género sexistas

MESOSISTEMA: entornos de socialización (escuela religión, comunidad, pares, relaciones sociales, ámbito laboral, afiliación politica, medios de comunicación, etc.) reproductores de estereotipos, prejuicios y roles sociales (sexistas, clasistas, racistas, etnocéntricas, homófobas, xenófobas, etc.)

EXOSISTEMA: sistemas estructurales (patriarcado, capitalismo, apartheid, globalizacion, heteronormatividad, nacionalismo, etc.)

MACROSISTEMA: ideologias (androcentrismo, clasismo, racismo, etnocentrismo, homofobia, xenofobia, etc.)

Fuente: elaboración propia

De acuerdo con la Figura 1, va a intentar explicarse la conformación de las denominadas "actitudes globales discriminatorias" (o "actitudes globales expropiatorias de derechos") teniendo en cuenta los diferentes entornos de desarrollo del sujeto. Se ha intentado mirar hacia el modelo ecológico del desarrollo humano por tres razones fundamentalmente: por un lado, porque los niveles establecidos por dicho modelo, en forma de entornos, permitiría operacionalizar el nivel de influencia de los elementos que perpetúan las desigualdades humanas (desde un nivel micro hasta un nivel macro) en el camino del desarrollo; por otro lado, este modelo describe el desarrollo desde un punto de vista psicosocial (aunque también pudiera interpretarse desde un punto de vista cultural y económico) y, por lo tanto, localiza la capacidad de desarrollo sobre las personas y su entorno y, por ende, también localizaría el poder de transformación de la realidad en las personas y su entorno (los sujetos dejan de ser una marioneta de la política económica para ser activos en su proceso de desenvolvimiento) y, por último, quizás dicho modelo pueda servir para aportar una respuesta tentativa acerca de por 
qué las desigualdades de género son la única constante manifiesta en todos los grupos de discusión realizados y por qué las mujeres sufren en mayor medida una serie de discriminaciones que "debieran” afectar de igual modo también a los hombres.

\section{a. El macrosistema del sujeto}

En el entorno relacionado con el macrosistema del sujeto se encontraría la visión (filosófica) del mundo androcéntrica, clasista, racista, etnocéntrica, homófoba o xenófoba. Es decir, una visión del mundo antiética y antimoral, en torno a la desigualdad, que localiza a los seres humanos en planos de oposición mediante la acción de discriminación como, por ejemplo, de las siguientes maneras:

- Desde el androcentrismo: "los hombres", como grupo social superiorizado, se sitúan como centro del mundo y "las mujeres", como grupo social inferiorizado, se sitúan en anexo a partir de "los hombres".

- Desde el clasismo: la "clase social capitalista" se presenta como grupo social dueño de la economía mundial y como grupo social superiorizado y la "clase social obrera", al servicio de las vicisitudes de la clase social capitalista, se sitúa como grupo social inferiorizado.

- Desde el racismo: se sitúa una supuesta "raza humana superiorizada" que se impone ante una supuesta "raza humana inferiorizada" desde el punto de vista moral, intelectual, físico, etc.

- Desde el etnocentrismo: un "grupo social étnico superiorizado" que se impone, principalmente desde el dominio del conocimiento (con el supuesto discernimiento de la verdad absoluta), a un grupo "social étnico inferiorizado".

- Desde la homofobia: un determinado grupo social, con una determinada orientación sexual confesada, se autolocaliza en un plano moral superiorizado y se opone a otros grupos sociales, con otras determinadas orientaciones sexuales, inferiorizados moralmente.

- Desde la xenofobia: un grupo social nacionalizado y superiorizado se opone a la inclusión de otros grupos sociales desnacionalizados (sujetos despojados de nación que constituyen el grupo de los apátridas o "sin papeles" del mundo) e inferiorizados. 
Desde el macrosistema del sujeto, una supuesta teoría del desarrollo del ser humano no discriminado (con un enfoque antiandrocéntrico, anticlasista, antirracista, antietnocéntrico, antihomófobo o antixenófobo) daría cuenta de qué tipo de desarrollo, es decir, qué tipo de visión filosófica (global) del desarrollo debería tenerse en cuenta para que el desenvolvimiento de las personas fuese ciudadanamente equitativo (D’Elia y Maingon, 2004).

\section{b. El exosistema del sujeto}

Por otro lado, en el entorno relacionado con el exosistema del sujeto, se encontrarían los sistemas (de carácter socioeconómico y político) sobre los que se conforman las ideologías anteriormente descritas y, al mismo tiempo, se les concede a éstas la capacidad estructural necesaria para su puesta en marcha como, por ejemplo, los siguientes casos:

- Desde el sistema patriarcal: se dictaminan las pautas de comportamiento de forma diferenciada y discriminatoria para hombres y mujeres. De esta manera, se coartan los derechos y libertades de las mujeres.

- Desde el sistema capitalista: se dictaminan, de forma discriminatoria, las funciones en la producción de bienes y servicios de la población en función de las clases sociales. De esta manera, se coartan las libertades del proletariado.

- Desde el sistema del apartheid o la esclavitud: se dictamina, de forma discriminatoria, la integridad moral (humana) de unos supuestos diferentes "grupos humanos raciales". De esta manera, se coartan las libertades y derechos de los "grupos raciales" inferiorizados.

- Desde el sistema de la globalización: se homogenizan los grupos étnicos tomando la modernidad occidental como molde para la homogenización etnocéntrica global. De esta manera, se coartan las libertades y derechos culturales de los grupos étnicos que intentan no ser homogenizados.

- Desde el sistema de la heteronormatividad: se desprecian, y en ocasiones se criminalizan, otras formas de orientación sexual distintas a la heterosexualidad (coartando las libertades y derechos sexuales de aquellos/as que no se manifiestan heterosexuales). 
- Desde el sistema del nacionalismo: se desprecia, y en ocasiones se criminaliza, al sujeto percibido como ajeno a la nación (coartando las libertades y derechos de aquella persona que es percibida, principalmente, como "extranjera sin papeles").

Desde el entorno del exosistema del sujeto, una supuesta teoría del desarrollo del ser humano no discriminado (antipatriarcal, anticapitalista, antiaparheid, antiglobalización, antiheteronormativa o antinacionalista) daría cuenta de qué estructura socioeconómica y política (global) permitiría el desenvolvimiento de dicho desarrollo desde el precepto de la equidad (justicia e igualdad). Es decir, este sistema constituye el entorno del desarrollo del ser humano en el que se deben adoptar normativas (globales), estrategias (globales) o políticas (globales) que propicien la configuración de un desenvolvimiento con dicha característica equitativa.

\section{c. El mesosistema del sujeto}

Asimismo, en el entorno relacionado con el mesosistema del sujeto (entorno social participativo del sujeto) se encontrarían los agentes e instituciones de socialización reproductores de los estereotipos y prejuicios en el imaginario colectivo de una determinada sociedad (sexista, clasista, racista, etnocéntrica, homófoba o xenófoba) tales como, por ejemplo: la escuela, la religión, la comunidad, los/as pares (amistades, compañeros/as, etc.), el ámbito laboral, el ámbito de afiliación política, los medios de comunicación, etc.

Desde el entorno del mesosistema del sujeto, una supuesta teoría del desarrollo del ser humano no discriminado (antisexistas, anticlasistas, antiracistas, antietnocéntrica, antihomófoba o antixenófoba) daría cuenta de cómo debieran pensar y actuar, psicosocioculturalmente, las personas (en el entorno social en el que habitan) para hacer factible una convivencia equitativa (basada en la justicia y la igualdad) en una comunidad ciudadana que aspira a ser global.

Además, se hace necesario tener en cuenta que los estereotipos y prejuicios pueden llegar a ocasionar, también, una distribución de los roles sociales (en función de la diferenciación entre grupos sociales previamente discriminados) establecidos para un supuesto "mantenimiento funcional de las sociedades". 


\section{d. El microsistema del sujeto}

Por último, en el entorno relacionado con el microsistema del sujeto (entorno familiar participativo del sujeto) se encontraría la familia, encargada de la atribución de roles de género sexistas para garantizar, supuestamente, su "autofuncionalidad", atribuyendo actividades diferenciadas para hombres y mujeres relacionadas con lo que "ellos deben hacer" y "ellas deben cumplir". Esa supuesta "autofuncionalidad" ha sido analizada desde el funcionalismo feminista por Miriam M. Johnson (1989) quien considera que el origen de la opresión de las mujeres por parte de los hombres reside en la configuración de la familia patriarcal.

Desde el entorno del microsistema del sujeto, una supuesta teoría del desarrollo del ser humano no discriminado daría cuenta de cómo debieran pensar y actuar, psicosocioculturalmente, las personas (en el entorno familiar) para llegar a concebir una atribución de roles de forma no segregacionista entre hombres y mujeres.

\section{Conclusiones}

Y aquí radica una pequeña explicación psicosocial de por qué las desigualdades de género son una constante manifiesta en todos los grupos de discusión realizados, por qué las discriminaciones que "debieran" afectar al mismo tiempo a hombres y mujeres resultan más evidentes en el discurso de las mujeres y, por lo tanto, por qué las mujeres se encuentran en una posición de desventaja en el camino hacia su desarrollo.

En primer lugar, la percepción de las discriminaciones mencionadas con anterioridad están sujetas a situaciones de conflicto (Chafetz, 1988; Morales y Páez, 1996; Verdú, 2013) derivadas de la interacción entre el grupo social inferiorizado y el grupo social superiorizado de tal forma que si no existiese una interacción entre ambos grupos, existiría una percepción basada en la igualdad entre las personas y no existiría, por lo tanto, un conflicto manifiesto en la vida cotidiana $\mathrm{y}$, consecuentemente también, no se percibiría dicha discriminación como tal (en primera persona). En este sentido, salvo raras excepciones, hombres y mujeres (socialmente configurados en sistemas patriarcales desde ideologías androcéntricas) están 
“obligados" a interaccionar en, prácticamente, todas las partes del mundo y, por lo tanto, existe un constante conflicto entre el grupo inferiorizado y el grupo superiorizado y es por ese motivo que podría percibirse la discriminación por razón de género en todos los grupos de discusión realizados independientemente del lugar de ejecución de los mismos.

En segundo lugar, también cabe preguntarse por qué no se perciben en un plano de igualdad, hombres y mujeres, independientemente del lugar de realización de los grupos de discusión. En este sentido, la discriminación por razón de género es el único proceso discriminatorio (inmerso en las "actitudes globales discriminatorias" o "actitudes expropiatorias de derecho") que actuaría de forma constante desde el microsistema del sujeto hasta el macrososistema del sujeto. En este sentido, el resto de procesos discriminatorios mencionados (basados en el clasismo, el racismo, el etnocentrismo, la homofobia o la xenofobia) se conforman desde el macrosistema, el exosistema y el mesositema del sujeto social pero no, aparentemente, desde el microsistema del sujeto social. Por lo tanto, teniendo en cuenta que la familia es el entorno de socialización más elemental, el proceso discriminatorio por razón de género acompaña al individuo desde su nacimiento, a través de la asignación de los roles de género sexistas, tal y como postulaban Candace West y Don H. Zimmerman (1987) y Janet Saltzman Chafetz (1992 [1989]). De hecho, las desigualdades de género derivadas de las diferencias en la distribución de los roles de género sexistas es una constante percibida por todos los grupos de discusión realizados independientemente del lugar de actuación de los mismos. Asimismo, cabe señalar que el agregado familiar también puede ser trasmisor de estereotipos y prejuicios, en el microsistema del sujeto, pero dichos estereotipos y prejuicios, en cualquier caso, obtienen el caldo de cultivo para su conformación en el mesosistema del sujeto.

Por último, tal y como propone Kimberlé Williams Crenshaw (1995), en ocasiones, la opresión vivida por las mujeres requiere, además de como elemento indispensable el análisis de la socialización de género, tener en cuenta otros aspectos de la socialización que también son discriminantes como pueden ser, por ejemplo, la "raza", la etnia, la edad, la orientación sexual, etc. Ahora bien, por qué dichas discriminaciones que "debieran" afectar al mismo tiempo a hombres y mujeres resultan más evidentes en el discurso de las mujeres participantes en los grupos de discusión que, respectivamente, en el discurso de los hombres. De forma hipotética, podría decirse que la carga de discriminación que sufren las mujeres es mayoritariamente más intensa que la discriminación que sufren los hombres por una cuestión "matemática" que tiene 
que ver con la suma de cargas discriminatorias partiendo del hecho de que "ellas" (las mujeres) sufren una discriminación conformada desde el microsistema del sujeto (a través de la atribución de roles de género sexistas) amén del resto de procesos discriminatorios que puedan ir sufriendo a partir del mesosistema, exosistema y macrosistema.

\section{BIBLIOGRAFÍA}

- Allport, Gordon Willard (1968 [1954]): La naturaleza del prejuicio (Trad. Cast. Ricardo Malfé). Buenos Aires: Educa.

- Amorós, Celia (1992): "Notas para una teoría nominalista del patriarcado". En: Asparkía. Investigación feminista, $\mathrm{n}^{\mathrm{o}}$ 1, pp. 41-58.

- Anderson, Benedict (1993 [1983]): Comunidades imaginadas. Reflexiones sobre el origen y la difusión del nacionalismo (Trad. Cast. Eduardo L. Suárez). México D.F.: Fondo de Cultura Económica.

- Anzaldúa, Gloria (2004 [1987]): "Movimientos de rebeldía y las culturas que traicionan". En: Gloria Anzaldúa, Kum-Kum Bhavnani, Avtar Brah, Margaret Coulson, bell hooks y Chela Sandoval: Otras inapropiables. Feminismos desde las fronteras (Trad. Cast. Rocío Macho, Hugo Romero, Álvaro Salcedo y María Serrano). Madrid: Traficantes de sueños, pp. 71-80.

- Barbera, Ester (2004): "Perspectiva cognitiva-social: estereotipos y esquemas de género". En: Ester Barbera e Isabel Martinez Benlloch (coords.): Psicología y género. Madrid: Prentice Hall, pp. 55-80.

- Beauvoir De, Simone (2008 [1949]): El segundo sexo (2 ${ }^{\text {a }}$ Edición, Trad. Cast. Alicia Martorrell). Madrid: Ediciones Cátedra.

- Bem, Sandra Lipsitz (1981): “Gender schema theory: A cognitive account of sex typing”. En: Psychological Review, vol. 88, nº 4, pp. 354-364.

- Benería, Lourdes y Sen, Gita (1983): "Desigualdades de clases y de género y el rol de la mujer en el desarrollo económico: implicaciones teóricas y prácticas”. En: Mientras Tanto, $\mathrm{n}^{\circ}$ 15, pp. 91-113. 
- Breckler, Stevem J. (1984): "Empirical validation of affect, behavior, and cognition as distinct components of attitude". En: Journal of Personality and Social Psychology, vol. 47, ${ }^{\circ}$ 6, pp.191-205.

- Bronfenbrenner, Urie (1987 [1979]): La ecología del desarrollo humano (Trad. Cast. Alejandra Devoto). Barcelona: Paidós.

- Butler, Judith (2006 [2004]): "Regulaciones de género". En: Revista de Estudios de Género. La ventana, $\mathrm{n}^{\circ} 23$, pp. 7-35.

- Chafetz, Janet Saltzman (1988): Feminist Sociology. An Overview of Contemporary Theories. Itasca, IL: F. E. Peacock Publishers.

- Chafetz, Janet Saltzman. (1992 [1989]): Equidad y Género. Una teoría integrada de estabilidad y cambio. Madrid: Ediciones Cátedras.

- Cobo, Rosa (2007): “Globalización y nuevas servidumbres de las mujeres”. En: Celia Amorós y Ana De Miguel (eds.): Teoría feminista: de la Ilustración a la globalización. De los debates sobre el género al multiculturalismo, Volúmen 3 (2 ${ }^{\circ}$ Edición). Madrid: Minerva Ediciones, pp. 265-300.

- Collins, Patricia Hill (2000): Black Feminist Thought. New York: Routledge.

- Crenshaw, Kimberlé Williams (1995): "Mapping the margins: Intersectionality, identity politics, and violence against women of color". En: Kimberlé Williams Crenshaw (ed.): Critical Race Theory: The Key Writings that Formed the Movement. New York: The New Press, pp. 357-583.

- Cruz De la, Carmen (2007): Género, Derechos y Desarrollo Humano. San Salvador: PNUD (Programa de Naciones Unidas para el Desarrollo). [en línea] Disponible en: http://www.otrodesarrollo.com/desarrollohumano/delaCruzGeneroDesarrolloHumano.pdf [0512-2011].

- D’Elia, Yolanda y Maingon, Thaís (2004): La equidad en el Desarrollo Humano: estudio conceptual desde el enfoque de igualdad y diversidad. Caracas: PNUD (Programa de Naciones Unidas para el Desarrollo).

- Davis, Angela (2005 [1981]): Mujeres, raza y clase (Trad. Cast. Ana Varela). Madrid: Ediciones Akal.

- Durán, María Ángeles (1993): "Introducción: La formación del pensamiento igualitario". En: María Ángeles Durán (coord.): Mujeres y hombres. La formación del pensamiento igualitario. Madrid: Castalia, pp.11-50. 
- Durán, María Ángeles (2012): El trabajo no remunerado en la economía global. Bilbao: Fundación BBVA.

- Espino, Alma (2007): “Género y pobreza: discusión conceptual y desafíos”. En: Revista de Estudios de Género. La ventana, $\mathrm{n}^{\mathrm{o}}$ 26, pp. 7-39.

- Fals Borda, Orlando (1959): Acción comunal en una vereda colombiana. Bogotá: Universidad Nacional de Colombia.

- Firestone, Shulamith (1976 [1970]): La dialéctica del sexo (Trad. Cast. Ramón Ribé). Barcelona: Kairós.

- Gilman, Charlotte Perkins (2011 [1911]): The Man-Made World or Our Androcentric Culture (Reimpreso). Auckland: The floating press.

- Hartmann, Heidi I. (1981): "The unhappy marriage of Marxism and feminism: towards a more progressive union". En: Lydia Sargent (ed.): Women \& Revolution. A discussion of the unhappy marriage of Marxism and Feminism. Québec: Black Rose Books, pp. 1-42.

- hooks, bell (1984): Feminist Theory: From Margin to Center. Cambridge: South End Press.

- Johnson, Miriam M. (1989): "Feminism and the theories of Talcott Parsons". En: Ruth A. Wallace (ed.): Feminism and Sociological Theory. Newbury Park CA.: Sage Publications, pp. 101-118.

- Lerner, Gerda (1990 [1986]): La creación del patriarcado (Trad. Cast. Mónica Tussell). Barcelona: Crítica.

- Millett, Kate (1970): Sexual Politics. New York: Doubleday.

- Morales, Francisco J. y Paez, Darío (1996): “Estereotipos, discriminación y relaciones intergrupos en España y en Latinoamérica”. En: Richard Yvon Bourhis y Jacques-Philippe Leyens (coords.): Estereotipos, discriminación y relaciones entre grupos. Madrid: McGrawHill Interamericana de España, pp.1-22.

- Nussbaum, Martha Craven (2000): Women and Human Development. The Capabilities Approach. Cambridge: Cambridge University Press.

- Organización de las Naciones Unidas (1996): Informe de la Cuarta Conferencia Mundial sobre la Mujer, Beijing, 4-15 de septiembre de 1995, A/CONF.177/20/Rev.1. Nueva York: ONU (Organización de las Naciones Unidas). [en línea] Disponible en: http://www.un.org/womenwatch/daw/beijing/pdf/Beijing\%20full\%20report\%20S.pdf $\quad$ [22-12$2010]$. 
- Ottosson, Daniel (2007): Homofobia de Estado. Un estudio mundial de las leyes que prohiben la actividad sexual con consentimiento entre personas adultas. Bruselas: ILGA (International Lesbian, Gay, Bisexual, Trans and Intersex Association). [en línea] Disponible en: http://ilga.org/historic/Statehomophobia/Homofobia_de_Estado_ILGA_07.pdf [12-052014].

- Paredes, Paloma (2012): "Pobreza al femenino: entre la perspectiva de género y el paradigma del desarrollo". En: Revista de Estudios de Género. La ventana, vol. IV, n 36, pp. 257-291.

- Programa de Naciones Unidas para el Desarrollo (1990): Informe sobre Desarrollo Humano 1990. Definición y medición del desarrollo humano. Nueva York: PNUD (Programa de Naciones Unidas para el Desarrollo). [en línea] Disponible en: http://hdr.undp.org/en/media/hdr_1990_es indice.pdf [29-08-2013].

- Sánchez Néstor, Martha (2005): “Mujeres indígenas en México: acción y pensamiento, Construyendo otras mujeres en nosotras mismas". En: Feminismos disidentes en América latina y el Caribe, Nouvelles Questions Féministes. Reveu internationale francophone, vol. 24, $\mathrm{n}^{\mathrm{o}} 2, \mathrm{pp} .41-53$.

- Sastre, Genoveva y Moreno, Monserrat (2004): "Una perspectiva de género sobre conflictos y violencia”. En: Ester Barbera e Isabel Martinez Benlloch (coords.): Psicología y género. Madrid: Prentice Hall, pp. 121-142.

- Schuler, Margaret (1997): "Los derechos de las mujeres son derechos humanos: la agenda internacional del empoderamiento". En: Magdalena León (comp.): Poder y empoderamiento de las mujeres. Bogotá: Tercer Mundo, pp. 29-54.

- Sen, Amartya (1985): Commodities and Capabilities. Oxford: North-Blackwell.

- Sen, Amartya (1990): "Gender and Cooperative Conflicts". En: Irene Tinker (ed.): Persistent Inequalities: Women and World Development. New York: Oxford University Press, pp.123-149.

- Sen, Amartya (2000 [1999]): Desarrollo y Libertad (Trad. Cast. Esther Rabasco y Luis Toharia). Barcelona: Planeta.

- Sen, Gita y Grow, Caren (1987): Development, Crises, and Alternative Visions: Third Word Women's Perspectives. New York: Monthly Review Press.

- Smith, Dorothy Edith (1974): "Women's Perspective as a Radical Critique of Sociology". En: Sociological Inquiry, n 44, pp.7-13. 
- Verdú, Ana Dolores (2013): “Género y conflicto en las relaciones de parejas heterosexuales: La desigualdad emocional". En: Cuestiones de género: de la igualdad y la diferencia, $\mathrm{n}^{\circ} 8$, pp. 165-181.

- Ward, Kathryn B. y Pyle, Jean Larson (1995): “Gender, Industrialization, and Development". En: Development, $\mathrm{n}^{\mathrm{o}}$ 1, pp. 67-71.

- West, Candace y Zimmerman, Don H. (1987): “Doing Gender”. En: Gender \& Society, vol. $1, \mathrm{n}^{\mathrm{o}} 2$, pp. $125-151$.

- Whitehead, Ann (2006 [1979]): "Some Preliminary Notes on the Subordination of Women”. En: IDS Bulletin, vol. 37, nº 4, pp. 24-27.

- Witting, Monique (2006 [1992]): El pensamiento heterosexual y otros ensayos (Trad. Cast. Javier Sáez y Paco Vidarte). Barcelona: Egales.

- Young, Kate (1988): “Introduction: reflections on meeting women's needs”. En: Kate Young (ed.): Women and Economic Development: Local, Regional and National Planning Strategies. Oxford: Berg Publishers, pp. 1-30.

- Young, Kimball (1967): “Concepto de actitud”. En: Kimball Young, John Carl Flügel, Gino Germani, Leo Kanner, Elizabeth B. Hurlock y Michael Argyle: Psicología de las actitudes (Trad. Cast. Eliseo Verón). Buenos Aires: Paidós, pp. 3-9. 